\title{
The needs assessment checklist: a clinical approach to measuring outcome
}

\author{
P Kennedy and LR Hamilton \\ Department of Clinical Psychology, National Spinal Injuries Centre, Stoke Mandeville Hospital NHS Trust, \\ Aylesbury, Bucks HP21 8AL, UK
}

\begin{abstract}
Objective: To evaluate the outcome of the Needs Assessment and Goal Planning Programme used in the rehabilitation of people with spinal cord injuries. The Needs Assessment Programme incorporates a behavioural indicator rating scale to detail the individual's progress and rehabilitation needs. This can also be used to evaluate the outcome of the rehabilitation programme in general. The Needs Assessment Checklist (NAC) forms part of the programme and is a tool which is used to evaluate rehabilitation outcome.

Setting: A purpose built, national spinal injuries centre in the United Kingdom.

Subjects: 82 patients who had completed the Needs Assessment Checklist, both at the beginning and towards the end of the rehabilitative process.

Results: Independence, as measured by the Checklist, was significantly greater in all domains at the time of the second Needs Assessment.

Conclusions: The Needs Assessment and Goal Planning Programme is successful in establishing greater client independence, whether assessed at a verbal or physical level. Team members have used the Needs Assessment Checklist as a behavioural indicator of rehabilitation outcome based on available standards of rehabilitative care. Further development of the Needs Assessment Checklist now needs to focus on establishing concurrent validity and test/retest reliability. The measure developed proved to be a useful, clinically relevant and patient friendly assessment of rehabilitation outcome.
\end{abstract}

Keywords: outcome measurement; needs assessment; goal planning; rehabilitation

\section{Introduction}

Research has shown that there is a discrepancy between what actually occurs and what people believe occurs during rehabilitation. ${ }^{1,2}$ Engagement in comprehensive rehabilitation has been found to be the best predictor of both post-discharge physical and psychological status, ${ }^{3}$ but adherence remains a problem across the healthcare domain. ${ }^{4}$ Slade $^{5}$ suggests that an assessment tool for everyday clinical use should go beyond symptomatology and behaviour observed by professionals, by incorporating the client's perceptions. McGrath, Marks and Davis ${ }^{6}$ found that by taking the client's wishes into account, compliance is certainly increased. Rehabilitation programmes aim to change behaviour in ways that support improved physical, social and psychological functioning, and outcomes are manifest changes of such programmes.

To manage the problems of institutionalisation and increase patient engagement, the Needs Assessment and Goal Planning Programme was implemented at the National Spinal Injuries Centre in 1989. ${ }^{1}$ Existing measures which assessed client ability were examined for suitability, including the FIM $^{7}$ measures of functional independence such as the Barthel Index, ${ }^{8}$

Correspondence: P Kennedy and measures of handicap such as the CHART. ${ }^{9}$ The FIM is a short (17 core item), easily administered, reliable and valid scale designed to measure the level of independence in self-care, sphincter control, transfers, locomotion, communication and social cognition. The Barthel Index was originally devised as a way of differentiating patients who were independent in activities of daily living (ADL), and includes the 10 most common areas considered within the broad definition of ADL. The Craig Handicap Assessment and Reporting Technique (CHART) is a weighted measure of function based on the World Health Organisation's conceptualisation of Impairment, Disability and Handicap. Dimensions include mobility, social integration and economic self-sufficiency. These measures are all well established with demonstrated reliability and validity properties and have been used extensively in health and rehabilitative care. $^{10}$

However, in general their brevity prevents a fine grain individual analysis of progress and they have been proved to be more relevant for assessing population samples. They possess noted floor and ceiling effects and are insensitive to small differences. ${ }^{10}$ Clinical team members often perceive these instru- 
ments to be population-focused and have little relevance to week-to-week individual patient-centred rehabilitation planning. Furthermore, they also have (apart from CHART) a dominant emphasis on physical restoration. It was felt by the authors of this paper that an assessment system was required which needed to be incorporated into the routine rehabilitation programme, was accepted by staff and easily integrated into clinical practice. Another key feature of the assessment measure required relates to the issue of independence. The purpose of rehabilitation is to enable people to regain control of their new needs and this can adequately be achieved by either verbal or physical means, thereby enabling all patients to achieve $100 \%$ of their goals, regardless of level of injury. The Needs Assessment developed helps the patient to think about personal needs and to increase both involvement in, and control over, the rehabilitative process. It helps staff understand what all the important issues are for a particular patient, and increases awareness and communication between the disciplines involved. ${ }^{10}$

In this study, the needs of the patient group were detailed, discussed and matched to service resource availability. They were then clustered into general domains and specific goals were generated for each need. The resulting checklist has nine specific rehabilitation domains, each with key behavioural indicators. These nine categories of need used in the checklist are: Activities of Daily Living; Skin Management; Bladder Management; Bowel Management; Wheelchair and Equipment; Mobility; Community Preparation; Discharge Coordination; and Psychological Issues. In the Checklist there are 216 behavioural indicators or quality standards. The Checklist is so detailed in order to find out exactly where rehabilitation attention needs to be focused, and to provide an indication of the existing skills that can be built upon.

In view of the conceptual confusion in rehabilitation, there is an arguable need to incorporate theory into evaluation from inception to provide a framework for treatment practices and integrating new information on methods. Treatment theory ${ }^{11}$ attempts to account for the processes which occur in the transformation from input to outcome. It begins by defining problems for a specific population and specifies: the critical input; the important steps to produce the desired effects; the mode of delivery; the expected outcome. This has been the framework for the Needs Assessment Programme.

The Needs Assessment has three conceptual levels. The first is the statement of need such as the general domains, for example Activities of Daily Living; the second level is the goal, for example being independent in food management; and the third level is the behavioural target, such as using feeding utensils independently at each meal time. A patient's performance is assessed in one of five categories of independence, ranging from total dependence to total independence, and includes a 'not applicable' category. No distinction is made between verbal and physical independence, enabling each patient to have the potential to achieve $100 \%$ independence.

The Needs Assessment Checklist is administered by an allocated keyworker with the patient, shortly after beginning the rehabilitation programme. Once completed, the category scores are converted into percentages representing 'Achieved', 'To be achieved' and 'Not applicable' scores. A graphical summary and an action plan detailing the areas of need are then produced. The scoring system shows strengths and needs in different aspects of functioning relative to one another so that priorities can be established. The keyworker coordinates the goal planning system with other members of the multi-disciplinary team and the patient to work on establishing and identifying needs, clarifying goals and specifying targets. Wright and Moffat ${ }^{12}$ highlighted that goals set should be specific and appropriate to everyday routines, and relevant to both the short-term and long-term needs of the patient. The Checklist is readministered when the patient is admitted to the pre-discharge ward, enabling comparison before and after goal planning to enable an evaluation of the individual's progress.

The Needs Assessment can be used to highlight broad indicators of rehabilitation outcome and is helpful for the organisation in planning of resources and the targeting of areas of weakness. It also acts as a demonstration to the individual patient of their specific needs, and matches their needs with available rehabilitative resources.

The purpose of this paper is to examine the outcome of rehabilitation. This was achieved by examining the areas of need as measured by the differences between the first and second Needs Assessment scores for a group of patients undergoing rehabilitation, with the specific hypothesis that need or independence is lower at the end of the rehabilitation programme.

\section{Method}

\section{Subjects}

Between July 1994 and December 1997, all 82 adults who had completed two Needs Assessments, the first within 2 weeks of being mobilised and the second upon transfer to the pre-discharge ward, were included in the study. The mean age of participants was 41 years, with a range between 16 and 74 . The group consisted of $72 \%$ male (59) and $28 \%$ female $(23)$. Fifty-four (66\%) had tetraplegic injuries and $28(34 \%)$ had paraplegic injuries. The mean time from admission to the first Needs Assessment was 92.5 days (range 6-245 days), and the mean time between the first and the second Needs Assessment was 95 days (range 17-235 days). 


\section{Materials}

The Needs Assessment, a behavioural checklist consisting of key indicators in the following domains: Activities of Daily Living (31 specific indicators); Skin Management (14 indicators); Bladder Management (12 indicators); Bowel Management (10 indicators); Wheelchair and Equipment (37 indicators); Mobility (20 indicators); Community Preparation (33 indicators); Discharge Coordination (34 indicators); and Psychological Issues (25 indicators).

\section{Procedure}

Shortly after the patient is mobilised a keyworker was allocated who had the responsibility of coordinating the Rehabilitation and Goal Planning Programme. The keyworker administered the Needs Assessment Checklist soon after mobilisation, which was then readministered when patients were admitted to the pre-discharge rehabilitation ward. For the purposes of this investigation, the 'To be achieved' scores were thought to be the best indicator of rehabilitation need. It is important to recognise that the Needs Assessment is the patient perception of their needs at the time of administration. The mean 'To be achieved' scores for the group were compared pre- and post-rehabilitation.

\section{Results}

The mean 'To be achieved' scores for the 82 patients in each of the nine domains were compared between the first and second Needs Assessments using the Wilcoxon Signed Rank Test. The mean scores, standard deviations, $\mathrm{Z}$ statistics and probability levels are presented in Table 1 .

It is clear that the Wilcoxon Signed Rank Test highlights a significant difference in the 'To be achieved' scores between the first and the second Needs Assessment on each domain, with the mean score being significantly lower on the second Needs Assessment. These results are presented graphically in Figure 1.

\section{Discussion}

The results from this review of 82 patients using the Needs Assessment Checklist are encouraging, but have to be treated with a certain caution. The weakness of this study is that the Needs Assessment, as a behavioural rehabilitation outcome measure, has not been checked for test/retest reliability, inter-rater reliability, concurrent validity or sensitivity to change. However, on a behavioural level, this study has shown that the needs of this patient group are significantly lower after rehabilitation. As there is no control comparison group, one can only speculate that the improvement is attributable to the Needs Assessment and Goal Planning Programme.

However, as this study is being based on treatment theory, ${ }^{11}$ it attempts to account for the processes that occur in the transformation from inputs into outcome. This is the first step in constructing a clinically relevant and sensitive outcome assessment measure. It is important when developing such measures to engage rehabilitation staff in the process and it is clear that this has been demonstrated by their completion of

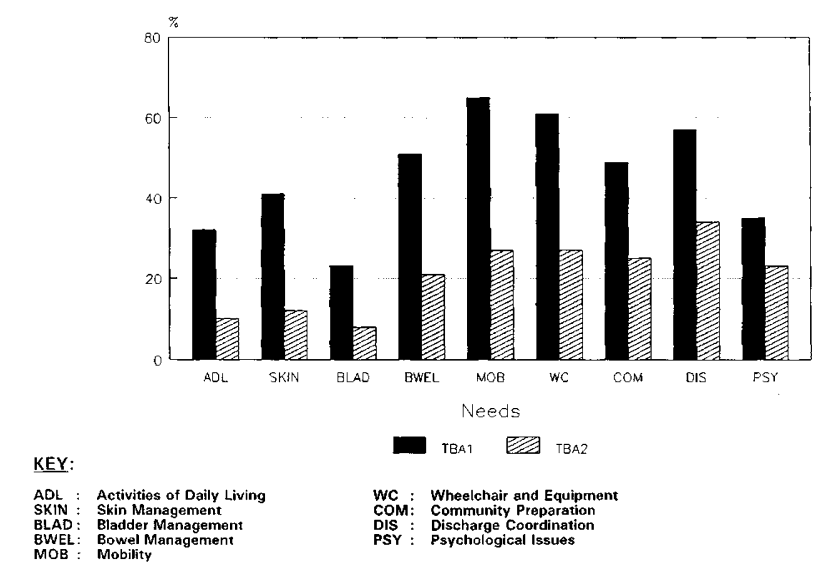

Figure 1 'To be achieved' mean scores: first and second needs assessments

Table 1 Means, standard deviations and Z statistics for the 'to be achieved scores'. $N=56$

\begin{tabular}{|c|c|c|c|c|}
\hline & $\begin{array}{c}T B A 1 \\
\text { Mean }(S D)\end{array}$ & $\begin{array}{c}T B A 2 \\
\text { Mean }(S D)\end{array}$ & $\begin{array}{c}Z \\
\text { Stat }\end{array}$ & $\begin{array}{l}\text { Prob. } \\
\text { level }\end{array}$ \\
\hline Activities of daily living & $32(26)$ & $10(17)$ & 7.1 & $* * * *$ \\
\hline Skin management & $41(27)$ & $12(15)$ & 6.9 & $* * * *$ \\
\hline Bladder management & $23(23)$ & $8(17)$ & 5.5 & $* * * *$ \\
\hline Bowel management & $51(31)$ & $21(25)$ & 5.9 & $* * * *$ \\
\hline Mobility & $65(24)$ & $27(21)$ & 7.7 & $* * * *$ \\
\hline Wheelchair and equipment & $61(22)$ & $27(20)$ & 7.2 & $* * * *$ \\
\hline Community preparation & $49(17)$ & $25(17)$ & 7.4 & $* * * *$ \\
\hline Discharge coordination & $57(23)$ & $34(22)$ & 6.5 & $* * * *$ \\
\hline Psychological issues & $35(16)$ & $23(14)$ & 5.4 & $* * * *$ \\
\hline
\end{tabular}

$* * * *=P<0.0001$ 
the Needs Assessment Checklist. It is now time to focus on developing the psychometric properties of the Needs Assessment Checklist with respect to reliability and validity. We also plan to assess concurrent validity by comparison with FIM scores.

The Needs Assessment and Goal Planning Programme has been shown to be successful in terms of its usefulness to staff, and in focusing the different team members on specific goals. ${ }^{1,13}$ MacLeod and MacLeod (1986) ${ }^{13}$ found that their goal planning system, which was based on the goal planning system set up in this study, was helpful in enabling patients to become more informed about the consequences of their injury and promote a sense of control over rehabilitation. Staff also found that goal planning was useful, helped with accountability and improved communication. It has been the experience of the authors that the goal planning Needs Assessment Checklist has been found to be a clinically appropriate measure which is popular with team members and that they report high satisfaction with its use. Essentially, it is a measure of health status and outcome from a patient-centred perspective as it concentrates on the patient's beliefs about their achievements. Moreover, the Checklist does cover the essential criteria used in the selection of the measure, ie that of providing the information that is needed. ${ }^{14}$ The Needs Assessment Checklist is a thorough measure which was matched with existing rehabilitative resources and focuses on the areas of need rather than deficiency, which contributes with the overall aim of providing the patient with a maximum sense of control and involvement in the rehabilitation process.

\section{Acknowledgements}

The authors would like to thank all the keyworkers and patients at the National Spinal Injuries Centre, Aylesbury, Buckinghamshire who kindly participated in this study.

\section{References}

1 Kennedy P, Walker L, White D. Ecological evaluation of goal planning and advocacy in a rehabilitative environment for spinal cord injured people. Paraplegia 1991; 29: 197-202.

2 Kennedy P, Fisher K, Pearson E. Ecological evaluation of a rehabilitative environment for spinal cord injured people: Behavioural mapping and feedback. British Journal of Clinical Psychology 1988; 27: 239-246.

3 Norris-Baker C, Stephens M, Rintala D. Patient behaviour as a predictor of outcome in spinal cord injury. Archives of Psychological and Medical Research 1981; 62: 602-608.

4 Kluss PA, Epstein LH. The measurement of medical compliance: a treatment of disease. In: P Karoly (ed.), Measurement strategies in health psychology. New York: Wiley.

5 Slade M. Needs Assessment. Involvement of staff and users will help to meet needs. British Journal of Psychiatry 1994; 165: 293 296.

6 McGrath JRM, Marks JA, Davis AM. Towards interdisciplinary rehabilitation: Further developments at Rivermead Rehabilitation Centre. Clinical Rehabilitation 1995; 9: 283 289.

7 Hamilton BB, Granger CV. Guide for the use of uniform data set for medical rehabilitation. Buffalo, NY: Research Foundation of State University of New York. 1990.

8 Mahoney FI, Barthel DW. Functional evaluation: The Barthel Index. Maryland State Medical Journal 1965; 14: 61 - 65 .

9 Whiteneck GG et al. Quantifying handicap: A new measure of long-term rehabilitation outcomes. Archives of Physical Medicine and Rehabilitation 1992; 73: 519-526.

10 Dittmars SS, Gresham GE (1997). Functional Assessment and Outcomes Measures for the Rehabilitation Health Professional. Gaithersburg, Maryland: Aspen Publications.

11 Keith RA, Lipsey MW. (1993). The role of theory in rehabilitation assessment, treatment and outcomes. In: Glueckauf RL, Sechrest LB, Bond GR, McDonel EC (eds). Improving Assessment in Rehabilitation and Health. London: Sage Publications.

12 Wright L, Moffat N. An evaluation of an individual programme planning system. The British Journal of Mental Subnormality 1992; 38: $87-93$.

13 MacLeod GM, MacLeod L. Evaluation of client and staff satisfaction with a goal planning project implemented with people with spinal cord injuries. Spinal Cord 1996; 34: 525-530.

14 Wade DT. Measurement in neurological rehabilitation. Oxford: Oxford University Press. 1992. 